\title{
Numerical study of the oscillations induced by shock/shock interaction in hypersonic double-wedge flows
}

\author{
Z. M. Hu • R. S. Myong - C. Wang • \\ T. H. Cho - Z. L. Jiang
}

Received: 30 September 2007 / Revised: 22 January 2008 / Accepted: 26 March 2008 / Published online: 18 April 2008

(C) Springer-Verlag 2008

\begin{abstract}
In this paper, the shock pattern oscillations induced by shock/shock interactions over double-wedge geometries in hypersonic flows were studied numerically by solving 2D inviscid Euler equations for a multi-species system. Laminar viscous effects were considered in some cases. Temperature-dependent thermodynamic properties were employed in the state and energy equations for consideration of the distinct change of the thermodynamic state. It was shown that the oscillation results in high-frequency fluctuations of heating and pressure loads over wedge surfaces. In a case with a relatively lower free-stream Mach number, the shock/shock interaction structure maintains a seven-shock configuration during the entire oscillation process. On the other hand, the oscillation is accompanied by a transition between a six-shock configuration (regular interaction) and a seven-shock configuration (Mach interaction) in a case with a higher free-stream Mach number. Numerical results also indicate that the critical wedge angle for the transition from a steady to an oscillation solution is higher compared to the corresponding value in earlier numerical research in which the perfect diatomic gas model was used.
\end{abstract}

Keywords Shock/shock interaction - Oscillation · Hypersonic · Double-wedge $\cdot$ Numerical study

Communicated by R. R. Boyce.

Z. M. Hu · R. S. Myong $(\bowtie) \cdot$ T. H. Cho

Research Center for Aircraft Parts Technology,

School of Mechanical and Aerospace Engineering,

Gyeongsang National University, Jinju 660-701, South Korea

e-mail: myong@gnu.ac.kr

Z. M. Hu

e-mail: bighumin@126.com

C. Wang $\cdot$ Z. L. Jiang

LHD, Institute of Mechanics, Beijing 100080, China
PACS $47.40 . \mathrm{Ki} \cdot 47.40 . \mathrm{Nm}$

\section{Introduction}

Shock/shock interactions in hypersonic flows can induce extremely high heating and pressure loads on the surface of an aircraft. Within the human's attempts to manned hypersonic flight, for example, unforeseen shock interactions caused airframe damage to an X-15. In February 1, 2003, damage to the thermal protection system (TPS) led to a structural failure in the left wing and the ultimate disaggregation of the USA space shuttle upon its re-entry. During this accident, shock/shock interaction was likely to be one of the factors that had caused the vulnerability of the aircraft. Shock/shock interactions are also important and fundamental issues occurring in the inlet flows of a hypersonic air-breath Scramjet with a multi-ramp shock-compression-driven intake. Damage to the ventral fin of the X-15 caused by shock/shock interaction phenomena was defined as one of unknown unknowns by Bertin and Cummings in $[1,2]$ in the development of hypersonic vehicles before the $\mathrm{X}-15 \mathrm{~A}$ accident. Unknown unknowns can have drastic consequences on the survival of the vehicle or the crew and lead to unacceptable increases in the costs of developing the vehicles [1]. As one of the key research issues in this area, numerous studies of shock/shock interactions can be found in literature [3-8].

Edney [3] used shock polar diagrams and classified the interactions of oblique shock waves and bow shocks on a cylinder. Through his experimental research, it was realized that abnormally high heating and pressure loads can be induced by shock/shock interactions on hypersonic vehicles and small changes in the geometry can lead to large changes in the overall flow structure. Olejniczak et al. [4] numerically studied shock interactions on double-wedge-like geometries. 
They found that there is a critical Mach number above which an underexpanded jet flow always occurs along the wedge surface. The interaction of the jet with the adjacent subsonic region results in large-amplitude pressure variations on the wedge surface. Recently, Ben-Dor et al. [5] revealed that a hysteresis and self-induced oscillations exist in the shock flow pattern for various angles of inclination of the second wedge. PIV and Schlieren visualization techniques were used to study the Mach 7 double-wedge flows [6,7]. It was found that the separation shock and bow shock show unsteady movements. The authors suggested that the motion of the bow shock is associated with unsteady phenomena in the reattachment region [7].

In Olejniczak's work [4], the simulations missed the oscillation phenomenon as it only concerned the steady-state solution. Ben-Dor's results [5] are based on an ideal fluid flow model. However, intense variation of temperature occurs in the shocked flowfields of interest. It is well known that thermodynamic properties in this condition will depend strongly on the gas temperature and can no longer be treated as constants. For example, the specific heat ratio of oxygen will decrease from 1.4 to 1.26 as the temperature increases from 300 to $3,000 \mathrm{~K}$. It should be noted that the specific heat ratio is one of the similarity parameters in the concerned flow problems. The lower and upper triple points propagate out of step with each other, and communicate reciprocally through the contact surface, a shear layer [5]. As the shear layer serves as an "intelligencer" during the oscillation process, the question arises of whether viscous dissipation can damp down the oscillation of shock wave flow patterns. In this paper, the oscillations induced by shock/shock interactions over double-wedge geometries in hypersonic flows were numerically studied by solving the $2 \mathrm{D}$ inviscid Euler equations for a multi-species system. Laminar viscous effects were additionally considered in some cases. Temperature-dependent thermodynamic properties were employed in the state and energy equations for consideration of the distinct changes of the thermodynamic state.

\section{Governing equations and numerical methods}

In 2D Cartesian coordinates, the conservative laws for multispecies, with the laminar viscous terms, are written as

$\frac{\partial U}{\partial t}+\frac{\partial F_{i}}{\partial x_{i}}=\operatorname{vis} \frac{\partial D_{i}}{\partial x_{i}}$

Here, $i=1,2$, and vis $=0,1$ for inviscid and laminar viscous flows, respectively. $U$ is the unknown variable vector, $F_{i}$ represents the inviscid flux vectors in the $x$ and $y$ directions, $D_{i}$ denotes the diffusion flux vectors, where

$$
\begin{aligned}
U= & {\left[\rho_{1}, \ldots, \rho_{n s}, \rho u_{1}, \rho u_{2}, \rho e\right] } \\
F_{i}= & {\left[\rho_{1} u_{i}, \ldots, \rho_{n s} u_{i}, \rho u_{i} u_{1}+p \delta_{i 1},\right.} \\
& \left.\rho u_{i} u_{2}+p \delta_{i 2},(\rho e+p) u_{i}\right] \\
D_{i}= & {\left[0, \ldots, 0, \tau_{1 i}, \tau_{2 i}, u_{j} \tau_{i j}+q_{i}\right], \quad j=1,2 }
\end{aligned}
$$

In the above equations, the total density of the mixture $\rho=\sum_{s p=1}^{n s} \rho_{s p}$, where $\rho_{s p}$ denotes the partial density of the species $s p . u_{i}(i=1,2)$ are the velocity components in the $x, y$ directions. $e$ and $p$ denote the total energy and the pressure of the mixture. $\tau$ and $q$ define the viscous stress and heat conduction terms. The transport properties of each pure component including the viscosity and thermal conductivity, and thermodynamic properties such as the special heat capacity and enthalpy, are obtained from relations given in the power series form of the temperature.

The total energy density is defined as

$\rho e=\rho h-p+\frac{1}{2} \rho u_{i} u_{i} \quad(i=1,2)$

Each species is usually assumed to be a thermally perfect gas, and the specific heat, enthalpy and entropy for each species can be calculated by the thermal polynomial equations

$$
\begin{aligned}
\frac{C p_{i}}{R_{i}}= & a_{1 i} T^{-2}+a_{2 i} T^{-1}+a_{3 i}+a_{4 i} T+a_{5 i} T^{2} \\
& +a_{6 i} T^{3}+a_{7 i} T^{4}+a_{8 i} \frac{1}{T^{3}} \\
\frac{h_{i}}{R_{i} T}= & a_{1 i} \frac{1}{T^{2}}+a_{2 i} \frac{1}{T} \ln T+a_{3 i}+\frac{a_{4 i}}{2} T+\frac{a_{5 i}}{3} T^{2} \\
& +\frac{a_{6 i}}{4} T^{3}+\frac{a_{7 i}}{5} T^{4}-\frac{a_{8 i}}{2} \frac{1}{T^{3}}+a_{9 i} \frac{1}{T}
\end{aligned}
$$

The appropriate constants in the above two equations can be found in the relevant literature [9]. Therefore, the enthalpy of the mixture, $h$, is obtained by summing the enthalpies of all species

$h=\sum_{i=1}^{n s} c_{i} h_{i}$

where $c_{i}$ is the mass fraction of species $i$ within the mixture. According to Dalton's law, the pressure $p$ is the sum of the partial pressure of each species

$p=\sum_{i=1}^{n s} \rho_{i} R_{i} T$

where $R_{i}$ is the gas constant of species $i$ and $T$ is the temperature of the gas mixture.

For the transport properties, the coefficients of thermal conductivity and the viscosity of the pure species can be approximated using the following equations

$\kappa=a+b T+c T^{2}+d T^{3}$

$\mu=A+B T+C T^{2}$ 
The appropriate constants in above two equations can be found in the relevant literature [10]. The transport parameters $\eta(\kappa, \mu)$ of the mixture are given by Wilkes' equations

$\eta_{\text {mix }}=\sum_{i=1}^{n s} \frac{X_{i} \eta_{i}}{\sum_{j=1}^{n s} X_{j} \phi_{i j}}$

$\phi_{i j}=\left[1+\left(\frac{\eta_{i}}{\eta_{j}}\right)^{0.5}\left(\frac{w_{j}}{w_{i}}\right)^{0.25}\right]^{2} /\left[8\left(1+\frac{w_{i}}{w_{j}}\right)\right]^{0.5}$

where the molecular weight and the molar fraction of each species are denoted by $w_{i}$ and $X_{i}$, respectively.

In [5], a W-modification of Godunov's scheme that has second-order accuracies both in space and time was used to solve the 2-D Euler equations for an inviscid perfect diatomic gas $(\gamma=1.4)$. An incorporation using of standard and diagonal stencils was performed to damp the numerical oscillations that might occur across strong shock waves in stationary flows. For numerical algorithms in the present study, the convective terms were discretized using the second-order DCD (dispersion controlled dissipative) scheme proposed by Jiang et al. [11,12] for capturing shock waves. The principle of DCD scheme aims at removing nonphysical oscillation by making use of dispersion characteristics instead of adding artificial viscosity. The DCD scheme has been well verified and validated during more than ten years applications into both inert [13-16] and chemically reacting flows [17-19]. In cases concerning laminar viscous effects, the dissipative terms were discretized by a fourth-order centered difference scheme. A third-order Runge-Kutta algorithm was used for time marching. The computational time step was globally determined by the CFL condition and varies from about $1.0 \times 10^{-5}$ to $1.6 \times 10^{-5}$ of the oscillation period. In the following simulations, a mixture of $4 N_{2}+O_{2}$ was used to approximate real air.

\section{Numerical results and discussion}

Figure 1 presents the computational domain for the doublewedge geometry in hypersonic flows. The shock wave interaction phenomena depend on the relevant parameters, which for inviscid flows are free stream Mach number $M_{\infty}$, the ratio of specific heats $\gamma$, the ratio of wedge lengths $L_{2}: L_{1}$, and the wedge angles $\theta_{1}$ and $\theta_{2}$. In the following simulations, $\gamma$ is not a constant as the thermodynamic properties are temperaturedependent. The length ratio $L_{2}: L_{1}=1: 2$, and $\theta_{1}=15^{\circ}$, while $\theta_{2}$ and $M_{\infty}$ change for different cases. Single-block structured grids with refinement near the wedge corner and surface were used to discretize all of the double-wedge geometries. The flow conditions were selected as $T_{\infty}=200 \mathrm{~K}$, and $p_{\infty}=9.66 \mathrm{kPa}$. Slip boundary condition was imposed on the wall in inviscid cases, while no-slip and adiabatic

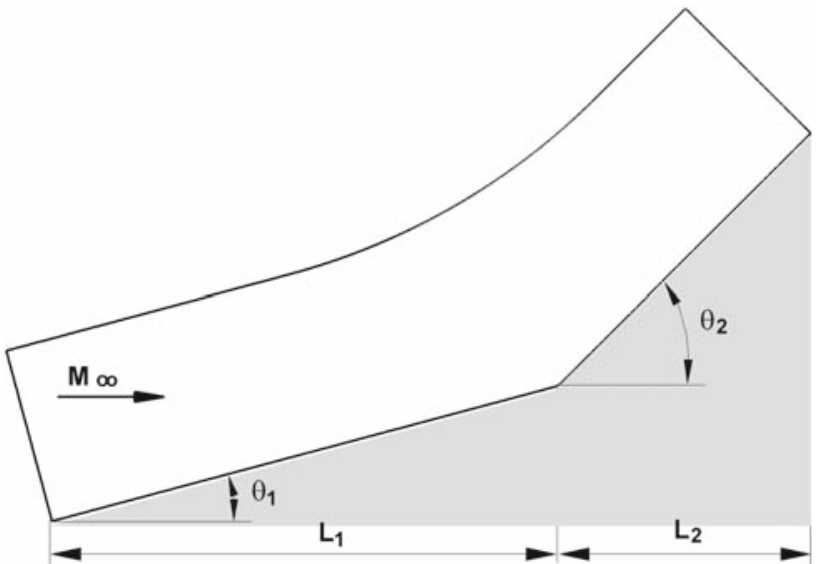

Fig. 1 Schematic of the computational domain for a double-wedge geometry in hypersonic flows

conditions were used for viscous cases. Supersonic inlet or outlet conditions were used for other computational boundaries.

\subsection{Fundamental flow features}

In a hypersonic flow, both wedges of a double-wedge geometry generate leading-edge shock waves, either oblique or curved. The resulting interactions between these shock waves can be classified according to Edney's scheme [3]. Olejniczak et al. [4] numerically studied Type VI, V, IV and IVr interactions as well as transitions among them. In the current study, only Type $\mathrm{V}$ interactions and possible oscillation phenomena were investigated.

Figure $2 \mathrm{a}-\mathrm{c}$ show three possible flow topologies, which depend on $\theta_{2}$, along with the notation for the constituent elements of the structure of the Type $\mathrm{V}$ interaction. The typical seven-shock structure, which is composed of three triple-shock sub-structures denoted by UTP, ITP, and LTP for concision, is given in (a). Each of these triple-shock structures contains three shock waves and a contact discontinuity surface. For example, the upper triple-point structure UTP includes (1) LSW-1, the leading-edge shock wave emanating from the first wedge; (2) BSW, the curved bow shock wave; (3) UMS, the Mach stem; and (4) UCS, the contact discontinuity surface emitting from the triple-point. Figure $2 b$ illustrates a six-shock structure which includes a triple-shock sub-structure and a regular interaction sub-structure. The flow pattern shown in Fig. 2c is nearly similar to that in (b), except that one of the refracted shocks of the regular interaction sub-structure undergoes a regular reflection from the second wedge surface. In Fig. 2a, the region bounded by two contact discontinuities, i.e. UCS and ICS, is termed a jet $[3,4]$. This region can be found clearly in [7] by the PIV visualization. A subsonic-supersonic transition process takes place in the flow region enclosed by the lower Mach stem LMS and 


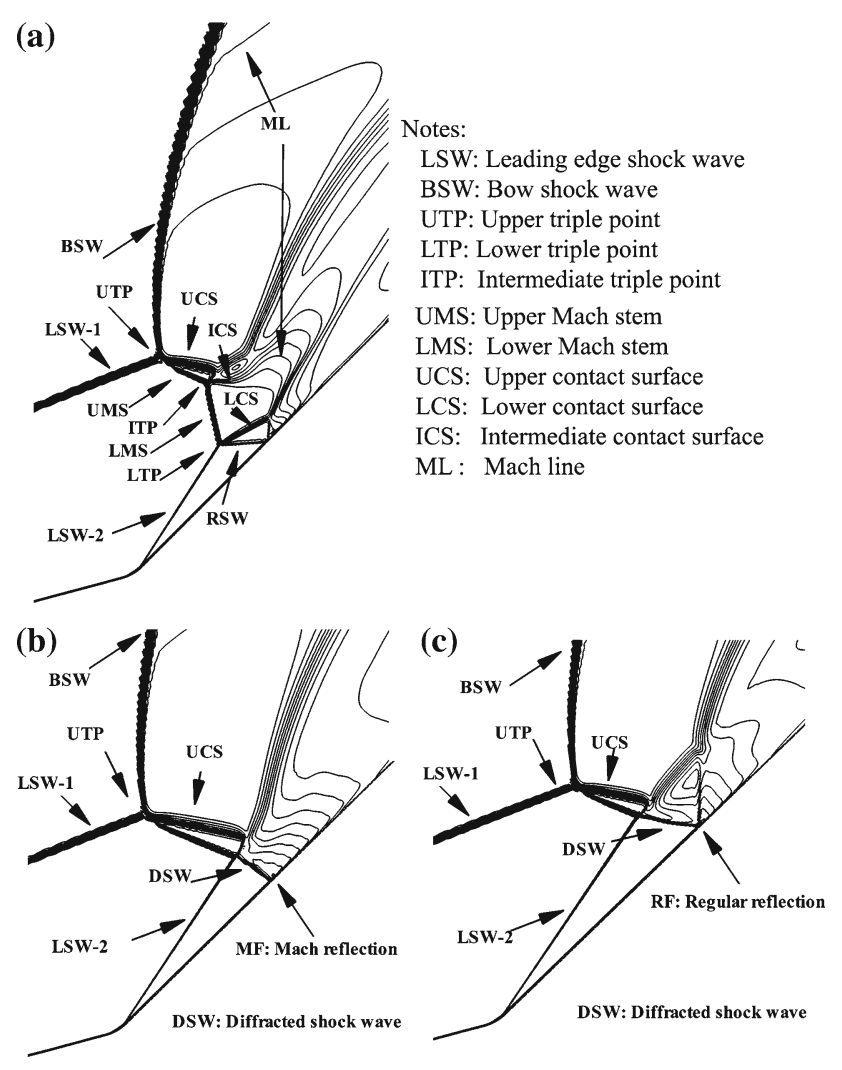

Fig. 2 Possible flow topologies for the Type V [3] shock/shock interaction, and notation for the flow structures (Mach number contours): a seven-shock configuration; b six-shock configuration with Mach reflection over the second wedge surface; c six-shock configuration with regular type of reflection over the second wedge surface

two contact discontinuities ICS and LCS experiences. This region appears as a convergent-divergent nozzle in which ICS and LCS act as nozzle blades. In the following subsections, it can be found that the three types of flow topologies mentioned above can successively take place during an oscillation period with a fixed $\theta_{2}$ value.

Fig. 3 Grid convergence (Mach number contours for a critical case: $M_{\infty}=7$, $\left.\theta_{1}=15^{\circ}, \theta_{2}=44.6^{\circ}\right)$

\subsection{Grid-convergence}

Figure $2 \mathrm{~b}$ presents a critical condition of a six-shock structure in which the reflected shock wave of LTP completely becomes a Mach stem. It will evolve into a seven-shock structure, as shown in Fig. 2a if $\theta_{2}$ increases slightly. This case was selected for the grid-convergence study. The flow conditions are $M_{\infty}=7, \theta_{1}=15^{\circ}$, and $\theta_{2}=44.6^{\circ}$. Three grids with different densities, Grid 1: $601 \times 151$, Grid 2: $701 \times 201$, and Grid 3: $801 \times 251$, were used to discretise the doublewedge geometry. The results and a comparison are presented in Fig. 3. They are in good agreement, although the shock thicknesses are somewhat different.

Starting from each steady solution shown in Fig. 3 as the initial condition, the above three grids were employed for an oscillation with the second wedge angle $\theta_{2}$ increased to $45^{\circ}$ while keeping $M_{\infty}$ and $\theta_{1}$ unchanged, respectively. In this case, the flow pattern of shock/shock interaction cannot keep steady any longer and transits into a periodic mode of shift between two seven-shock structures (see Fig. 2a) with different locations of interaction and lengths of Mach stem (LMS). The shock structures at the extreme left and right locations associated with each grid are shown in Fig. 4a. The details of the flow pattern and its evolution are illustrated in the following section. It can be seen that the shock flow patterns and the oscillations are well captured with Grid 2 and Grid 3, and they are fairly unique. However, Grid 1 doesn't function well as other two with respect to the resolutions for extreme locations and unsteadiness of the contact surface.

Figure $4 \mathrm{~b}$ presents profiles of non-dimensional pressure along the wedge surface associated with the extreme conditions for each grid resolution. The pressure is normalized by the pressure of free stream flow, $p_{\infty}$, and the location along the wedge surface is normalized by the first wedge length, $L_{1}$. In each profile, the first normalized pressure level corresponds to the pressure, $p_{1}$, behind the first leading shock,
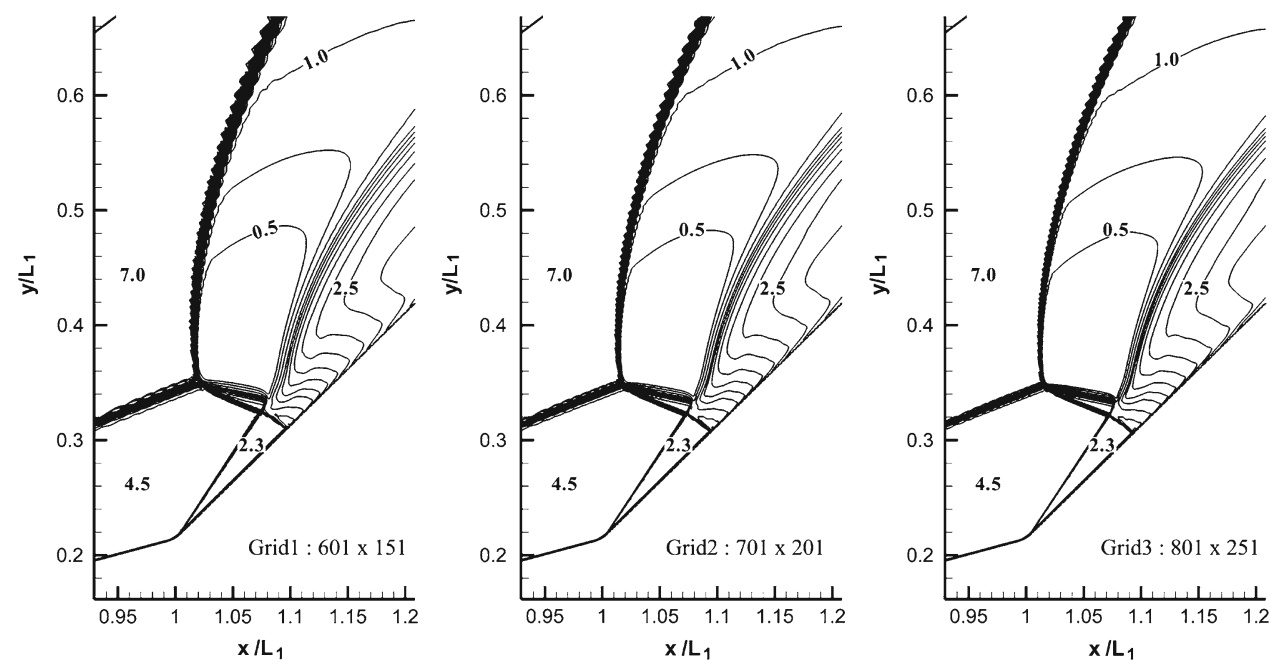

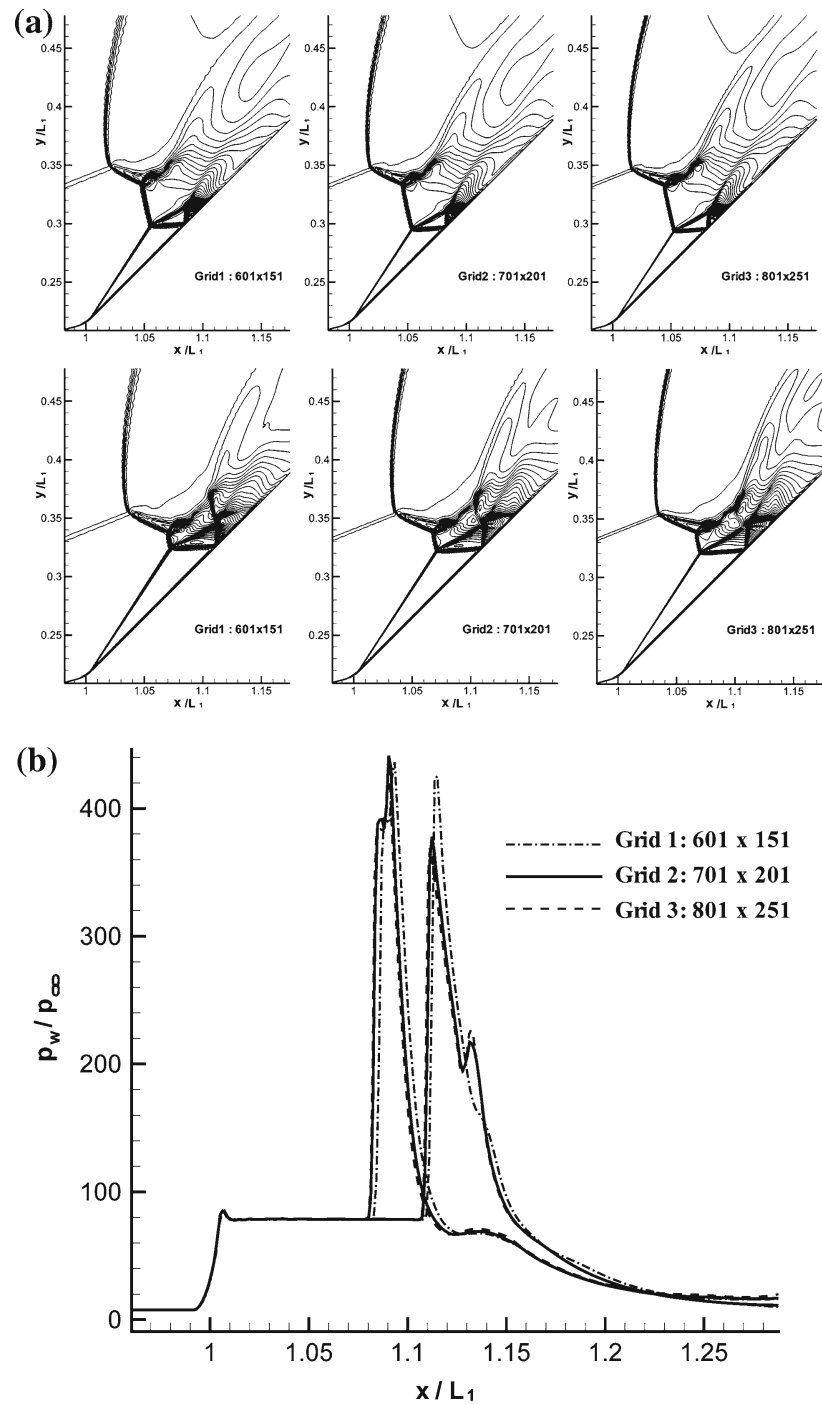

(c)

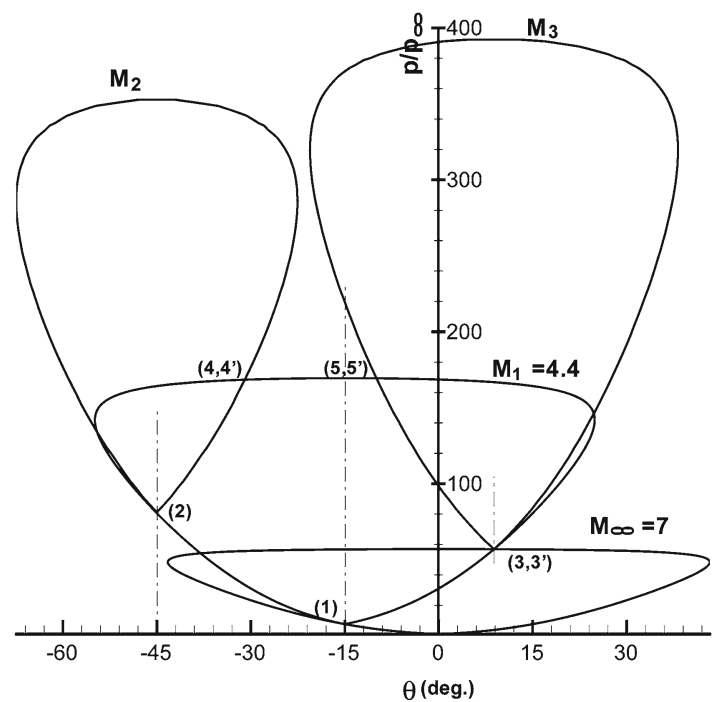

Fig. 4 Grid convergence for an oscillation case $\left(M_{\infty}=7, \theta_{1}=15^{\circ}\right.$, $\theta_{2}=45^{\circ}$ ): a shock patterns at extreme left and right locations shown by Isopycnics; $\mathbf{b}$ Pressure along the second wedge surface; $\mathbf{c}$ shock polar for an inviscid perfect diatomic gas $(\gamma=1.4)$
LSW-1, while the second level, $p_{2}$, is due to the second leading shock, LSW-2. It should be noted that during the oscillation processes, the local flow conditions behind these two oblique shock waves keep uniform and steady. Moreover, the temperature-dependency effect within these two regions is not too significant in this case with a moderate $M_{\infty}=7$ of the free stream flow. Therefore, the shock polar analysis (as shown in Fig. 4c for an inviscid perfect diatomic gas $(\gamma=1.4))$ can be used to approximately validate the numerical results. It is useful to present shock interaction flows using pressure-deflection or $(p-\theta)$ shock polar diagrams, in which the pressure jump across a shock wave is plotted versus the flow deflection angle. Following Edney's [3] angle convention, in Fig. $4 \mathrm{c}$ the positive angle corresponds to clockwise deflections from the horizontal. As shown by Fig. $4 \mathrm{c}$ the analytical pressure jumps are $p_{1} / p_{\infty}=7.56$ and $p_{2} / p_{\infty}=80.2$, while the corresponding ones from the numerical simulations on all grids mentioned above are $p_{1} / p_{\infty}=7.54$ and $p_{2} / p_{\infty}=78.6$ shown in Fig. 4b. The computation agrees well with the theory. Nevertheless, it is reasonable to observe a little bigger discrepancy of $p_{2} / p_{\infty}$ since the temperature-dependency effect in the flow domain behind the LSW-2 is a more significant than that in the flow domain behind LSW-1. Each pressure peak in Fig. $4 \mathrm{~b}$ results from the re-reflection over the second wedge surface of the reflected shock wave, RSW, of each lower triple point structures, LTP (see the notation in Fig. 2a). Due to unsteadiness, these maximum pressures cannot be obtained from shock polar analysis, neither can the flow conditions shortly behind the Mach stem, LMS. It can be seen from Fig. 4b that either the pressure profiles or the reflection points of RSW agree well with each other for solutions of Grid 2 and Grid 3, while those for Grid 1 show some differences. In the Grid 1 solution, the reflected shock of RSW over the wedge surface is stronger and the LCS is somewhat smeared and the reflected pressure of RSW over the wedge surface was consequently driven up. Hence, these facts may be used to provide the convergence of grid resolution in the present study. In [4], a grid of $1,024 \times 1,024$ was used for steady computation. Such a grid of high density is not affordable for the present unsteady computation. Since the mesh was clustered towards the wedge surface and the second wedge corner in the present study, most of the important flow features of interest can be captured. In the following simulations, the moderate grid with $701 \times 201$ nodes was therefore employed.

\subsection{Shock/shock interaction induced oscillations}

Successive isopycnic frames in Fig. 5 show the evolution of the shock configuration during two periods of oscillations. The flow conditions are $M_{\infty}=7, \theta_{1}=15^{\circ}$, and $\theta_{2}=45^{\circ}$. In this figure and the following ones, the frame numbers correspond to time sequences. For example, frames 470 to 
Fig. 5 Type V interaction and sequential isopycnics showing the oscillation of the interaction structures $\left(M_{\infty}=7\right.$, $\left.\theta_{1}=15^{\circ}, \theta_{2}=45^{\circ}\right)$

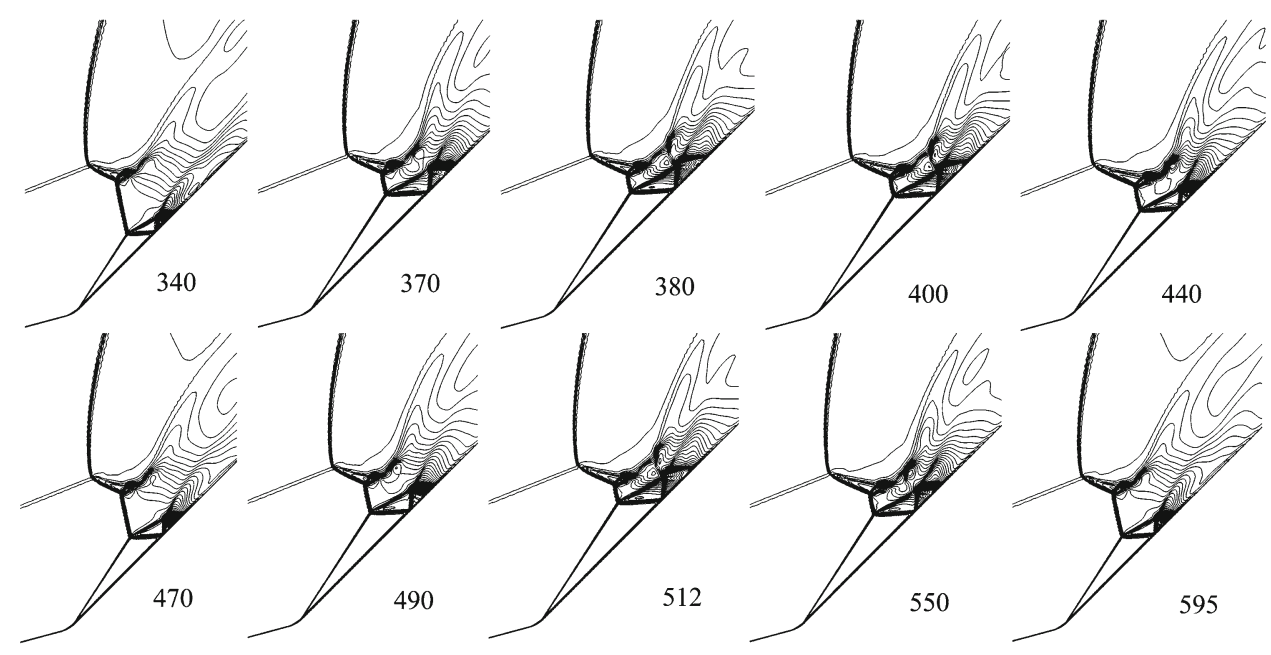

595 in Fig. 5 present the flow structures at five significant instants within one oscillation period with a time step equal to $1 / 125$ of the period. In frame 340 , LTP reaches its extreme left position and the Mach stem of LTP reaches its maximum length. It should be noted that this maximum length and the upstream location of the LTP can no longer appear during the following oscillation processes. From frame 340 to frame 370 , the LTP is seen to move downstream to its extreme right position at frame 380, where the Mach stem, LMS reaches its minimal length. Following frame 400, LMS moves upstream and begins to grow until it reaches its second maximum length at frame 470 . It can be seen clearly that the second maximum length of the LMS is shorter than the former maximum length given in frame 340. The first critical state in frame 340 is due to the 'overgrowth' of the lower triple-shock structure from the initial condition. In frames 380,400 and 440, the rapid shift in the position of the triple points causes instability of the contact surfaces of UTP and ITP. It should be noted that the flow structure in frame 380 and frame 512 are nearly identical, and the flow structure in frame 470 and frame 595 are nearly identical as well. It was reported in [5] that within an oscillation period, there are two different maximum lengths of the lower Mach stem, as well as two different minimum lengths. In the present study, there appears a unique maximum or minimum length during one oscillation period. A seven-shock to six-shock transition was also reported in [5], in which a different flow condition was set for the initiation, i.e. a perfect diatomic gas model $(\gamma=1.4)$ along with a higher free stream Mach number, $M_{\infty}=9$.

In order to reveal the cause of the difference mentioned above, a case with a higher free stream flow Mach number, $M_{\infty}=9$, was studied. Unfortunately, a stationary six-shock structure was obtained with the second wedge angle unchanged, $\theta_{2}=45^{\circ}$. It can be tentatively concluded that the flow model has a strong effect on the shock flow patterns and possible oscillation. Oscillation of shock flow pattern can be obtained when $\theta_{2}$ is increased to $46.4^{\circ}$. Figure 6 presents shock patterns at several important instants within one oscillation period with a time step equal to $1 / 210$ of the period. The oscillations in this case are accompanied by a transition to regular reflection, similar to what was explained in [5]. Frames 310 to 320 indicate the interaction between the leading edge shock wave(LSW-2) starting from the second wedge and the Mach stem (UMS) of the upper triple point (UTP) evolves into a Mach interaction from a regular interaction. The Mach stem (LMS) of the lower triple-wave structure reaches its left extreme position and maximum length in frame 320. Starting from frame 360, the lower triple point (LTP) moves downstream and reaches its right extreme position at frame 380 hereafter, it begins to travel upstream again towards another left extreme position at frame 415. During this oscillation sub-process, the lower Mach stem (LMS) always exists. However, the lengths of LMS at the former (in frame 320) and latter (in frame 415) extreme left position are remarkably different. The evolution to the first extreme left state appears similar to the "overgrowth" described in Fig. 5. In contrast, the "overgrowth" can be repeated in this case, as can be seen in subsequent frames. From frame 450 to frame 520, the Mach interaction becomes a regular interaction hereafter, it changes back into Mach interaction. Finally LTP reaches its first left extreme position at frame 530. The entire oscillation process, frames 320 to 530, then repeats periodically. Instability associated with UCS and ICS can be seen in several frames. Comparing Figs. 5 and 6, the oscillations of the shock flow pattern can be completely different if the free stream Mach number changes.

The out-of-step movements of the lower triple point (LTP) and the upper triple point (UTP) are crucial in the generation of the observed oscillations [5]. Figure 7a-d show several close-ups of the flow structure around the triple points at the transients when the lower triple point nearly reaches its extreme left or right locations. The numbers in the figures denote different time instants, corresponding to the frame 
Fig. 6 Type V interaction and sequential isopycnics showing the oscillation of the interaction structures $\left(M_{\infty}=9\right.$, $\left.\theta_{1}=15^{\circ}, \theta_{2}=46.4^{\circ}\right)$
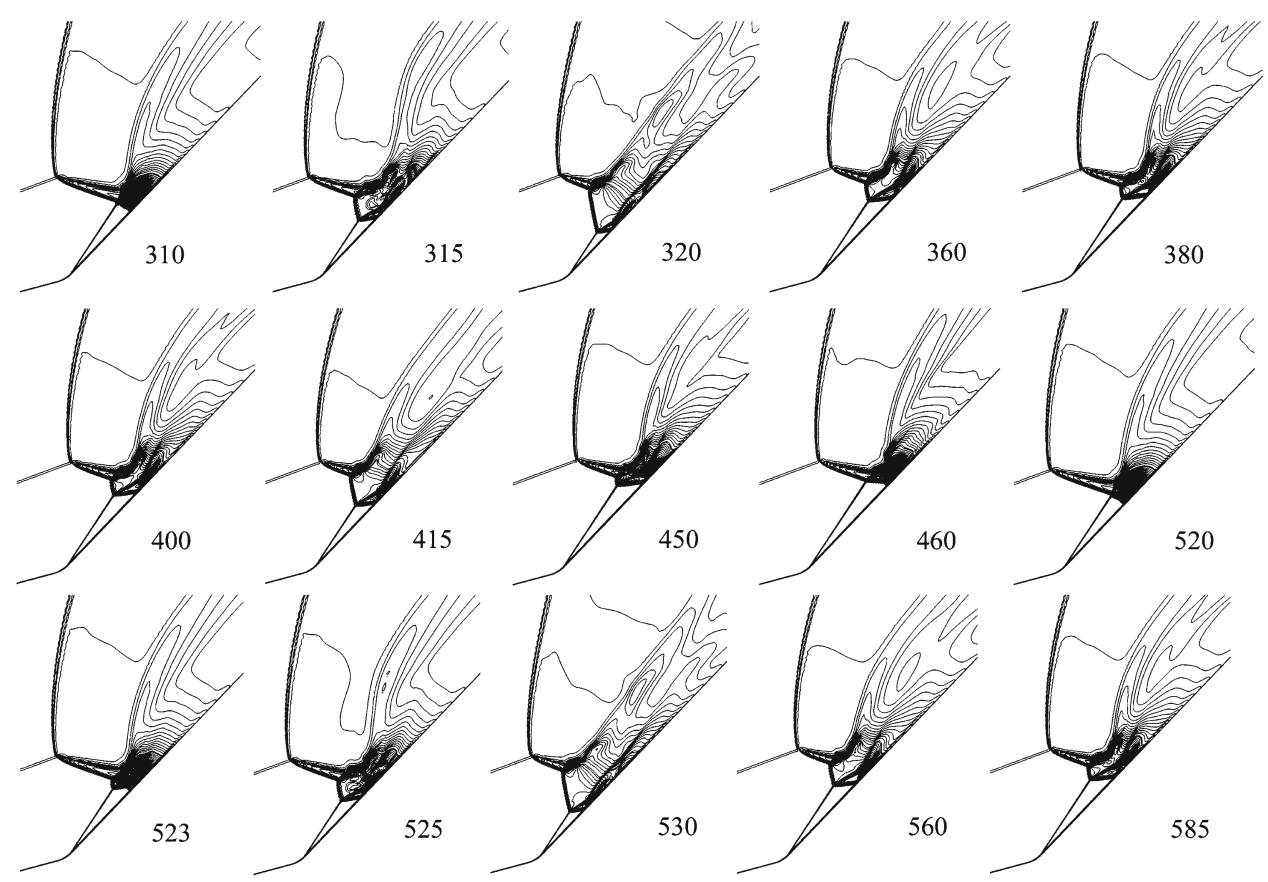

Fig. 7 Out-of-step movements of the lower and upper triple points shown by the isopycnics. a, b: $M_{\infty}=7, \theta_{2}=45^{\circ}$; c, d: $M_{\infty}=9, \theta_{2}=46.4^{\circ}$
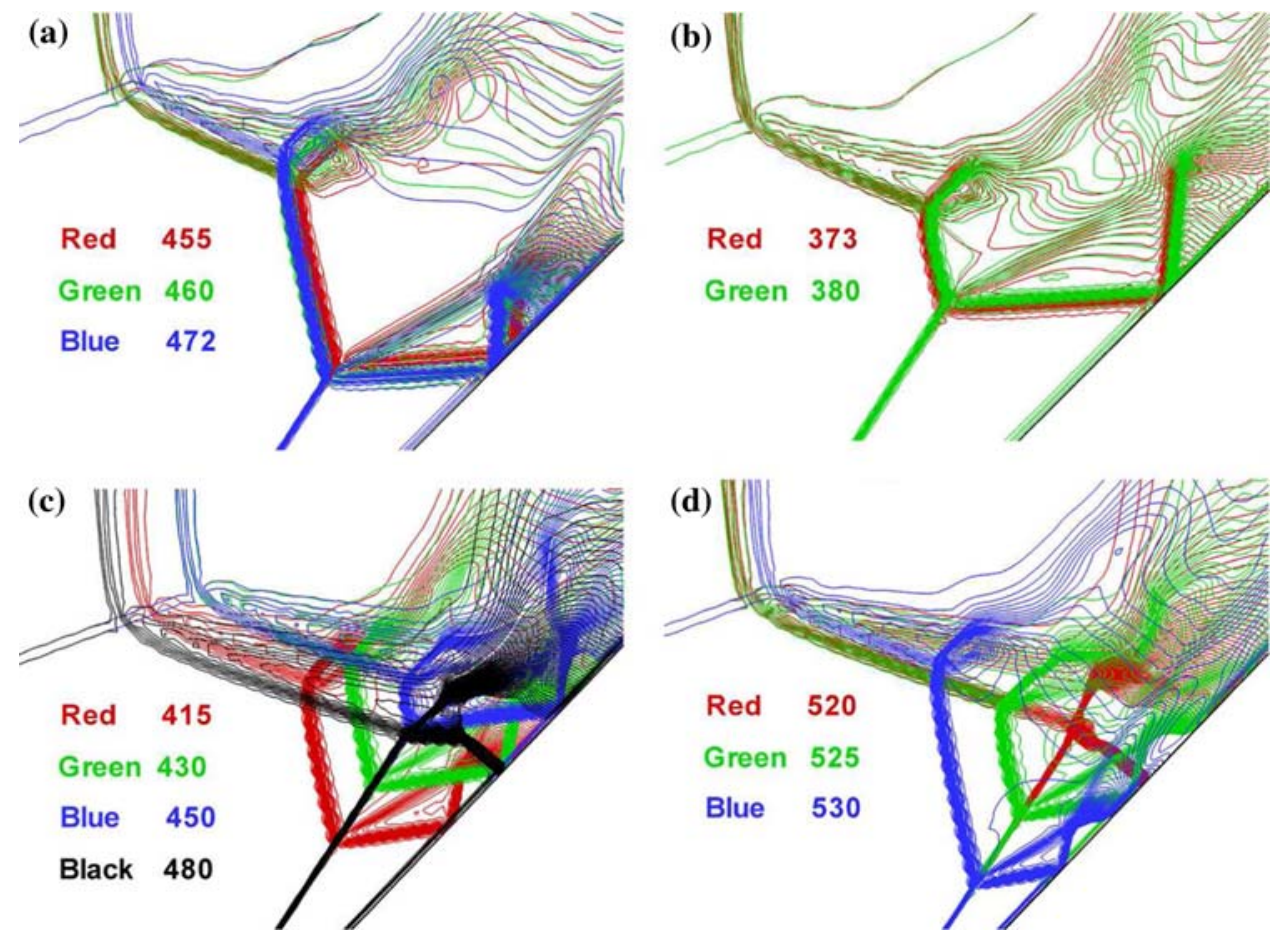

numbers in Figs. 5 and 6. As shown by the red density contour in (a), the upper triple point reaches its maximum left position at instant 455, and remains stationary until instant 460 , which implies that the pre- and post-shock flow parameters of UTP meet their matching conditions. However, the lower triple point together with the lower Mach stem continue to move in the left direction, as indicated by the green contour. The motion of the LTP with a small delay relative to that of the UTP changes the inclination angles of UCS and BSW.
Consequently, the former matching condition of UTP breaks down. Finally, at instant 472 when LTP reaches its left terminal, the upper triple point shown with the blue contour has started to move in the right direction and the oscillation is initiated. Figure $7 \mathrm{~b}$ shows the delay of the lower triple point motion when it arrives at its extreme right position. From Fig. 7a and $\mathrm{b}$ it can be seen that the shock angles of LSW-1 and LSW-2 never change during the oscillations, which is consistent with the conclusion of [7]. For the case 

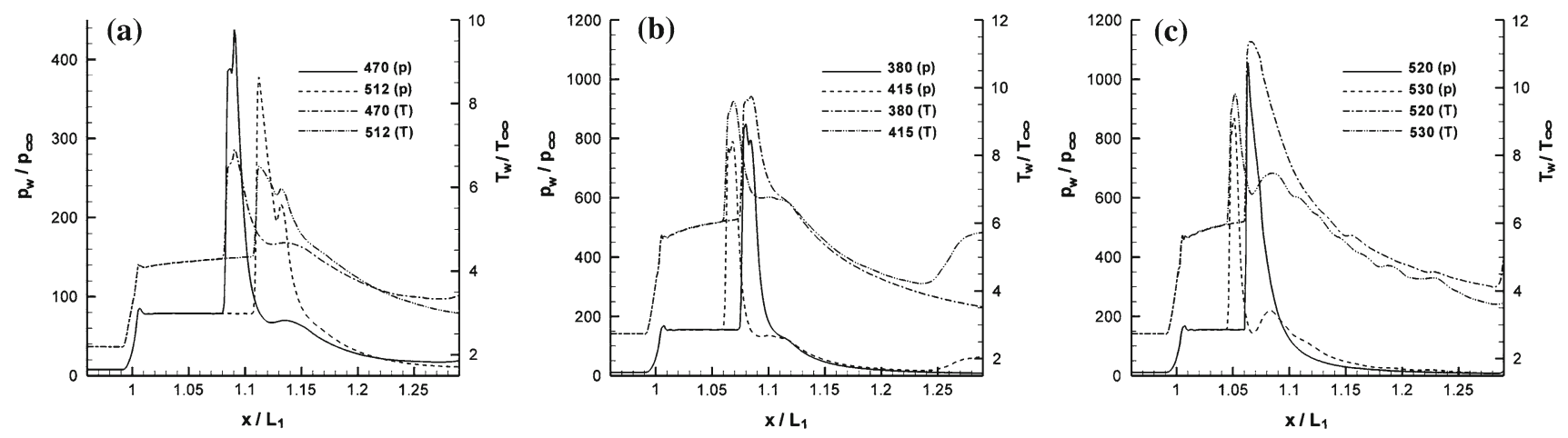

Fig. 8 Pressure and temperature distributions along the wall surface during the oscillation: a $M_{\infty}=7, \theta_{2}=45^{\circ} ; \mathbf{b}, \mathbf{c} M_{\infty}=9, \theta_{2}=46.4^{\circ}$

of $M_{\infty}=9$, Fig. 7c and d show the decline of LMS from the second maximum length and the regrowth of LMS into its first maximum length, respectively. As illustrated by the red and blue contours in (c) or by the red and green contours in (d), the delay of LTP is more distinct than that in the case with a lower free stream Mach number $M_{\infty}=7$.

Type $\mathrm{V}$ shock/shock interaction can induce high pressure and heating loads on the second wedge surface due to shock wave reflection. It should be noted that the observed oscillation of multi-shock structures causes vibration in the pressure and heating loads. As shown in Fig. 8a, the maximum wall pressure in the case of $M_{\infty}=7$ varies from approximately $440 p_{\infty}$ at the extreme left position (frame 470 in Fig. 5) to $380 p_{\infty}$ at the extreme right position (frame 512 in Fig. 5). In contrast, the fluctuation amplitude of the maximum wall temperature is about half of the free stream temperature during an oscillation cycle. Therefore, the oscillation of the triple points results in more severe vibration of aerodynamic force than heating load along the wedge surface in a lower $M_{\infty}$ case. The same phenomena can be found in Fig. $8 \mathrm{~b}$ during the sub-cycle of oscillation (frames 380 to 415 in Fig. 6) without transition from a seven-shock configuration into a six-shock structure for the case when $M_{\infty}=9$. However, as can be seen in Fig. 8c, the second wedge surface experiences high loads of both heating and force and their severe fluctuations during the sub-cycle of oscillation accompanied by the transition from a six-shock to a seven-shock configuration. The pressure and temperature after the Mach reflection of the DSW (frame 520 in Fig. 6) become nearly $1,080 p_{\infty}$ and $11 T_{\infty}$, respectively. The values represent a 30 and $20 \%$ increase, respectively, over those surface loads when LTP reaches its extreme left position (frame 530 in Fig. 6). Nevertheless, much higher pressure pulse can be achieved on the second wedge surface during the transition between a six-shock and a seven-shock flow patterns. Figure 9 shows the historical records, within two oscillation periods, of the maximum pressure along the second wedge surface and the corresponding position where the maximum pressure occurs as a result of the shock wave reflection of the RSW or DSW. In this figure, curve ABC

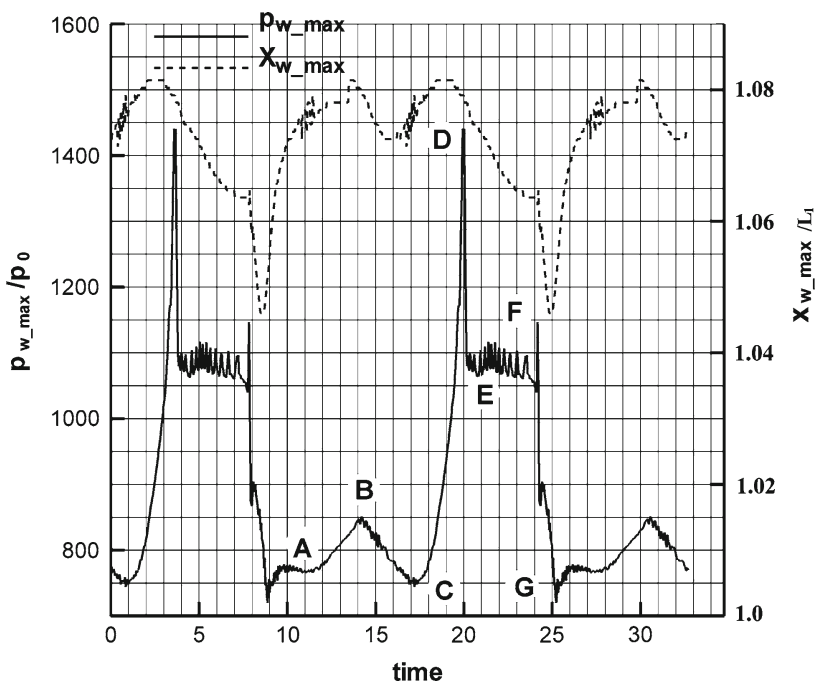

Fig. 9 Variation of the maximum pressure along the wedge surface (solid line) and its location (dash line) $\left(M_{\infty}=9, \theta_{1}=15^{\circ}, \theta_{2}=46.4^{\circ}\right)$

corresponds to the sub-cycle of oscillation between two different seven-shock configurations mentioned above, while curve EF presents the oscillation stage during which a sixshock configuration always exists and moves along the wedge surface. The highest pressure pulse, which is around $1,440 p_{\infty}$, in the vicinity of point $\mathrm{D}$ occurs shortly before the shock pattern transits from a seven-shock to a six-shock configuration. Another relatively lower pressure pulse around point $\mathrm{F}$ in Fig. 9 appears shortly after the shock pattern shifts from a six-shock to a seven-shock configuration.

Further simulations when $M_{\infty}=7$ and $\theta_{1}=15^{\circ}$ indicate that the oscillations occur within a small scope of $\theta_{2}$. A Type VI-Type V interaction transition occurs at $\theta_{2}=41.5^{\circ}$. From $\theta_{2}=41.5^{\circ}$ to $\theta_{2}=44.6^{\circ}$, steady solutions with six-shock interaction configurations (as shown by Fig. 2c) were obtained. Above $\theta_{2}=46^{\circ}$, the solutions converge to Type V flow topologies with seven-shock configurations which is defined in Fig. 2a. It was found that the oscillations take place within the angle range $\theta_{2}=44.8^{\circ}$ to $45.9^{\circ}$. Seven-shock 


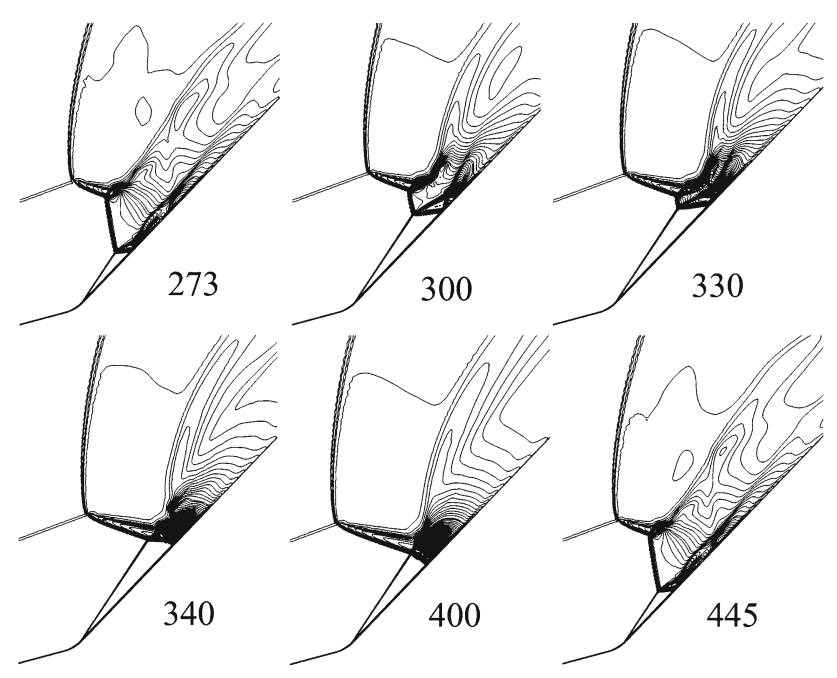

Fig. 10 Type $\mathrm{V}$ interaction and sequential isopycnics showing the oscillation of the interaction structures $\left(M_{\infty}=9, \theta_{1}=15^{\circ}, \theta_{2}=\right.$ $\left.46.2^{\circ}\right)$

configurations always exist during these oscillations. It was found that the highest pressure load associated with the type $\mathrm{V}$ interaction in this case occurs within $\theta_{2}=41.5^{\circ}$ to $44^{\circ}$; in other words, steady six-shock configurations generate higher surface loads compared to seven-shock structures.

When $M_{\infty}=9$, much higher loads with sharp fluctuations impose on the wedge surface due to a shock wave reflection from the second wedge. Further studies show that oscillations can be triggered within an approximate range of $\theta_{2}=46.2^{\circ}$ to $46.8^{\circ}$, which differs from the oscillation range, $\theta_{2}=42^{\circ}$ to $43^{\circ}$, obtained in [5]. Numerical results also show that the oscillation processes differ from each other with different values of $\theta_{2}$. For a case with a relatively smaller angle, $\theta_{2}=46.2^{\circ}$, Figure 10 shows the sequential isopycnics within one oscillation cycle. The evolution of shock structures is simpler than that at $\theta_{2}=46.4^{\circ}$. The flow topology directly transits between seven-shock and six-shock configurations, without the sub-oscillation illustrated by frames 380 to 415 in Fig. 6. For cases with angles above $\theta_{2}=46.6^{\circ}$, identical flow evolution to that shown in Fig. 5 is obtained, in which the seven-shock structure always exists during the oscillations. The aforementioned phenomena dependent on the wedge angle differ from the numerical results of [5]. This difference is likely to be associated with the temperaturedependent thermodynamic properties incorporated in this study.

\subsection{Ideal gas model}

In this section, the unsteady flow associated with the shock/ shock interaction induced oscillation for an inviscid perfect diatomic gas $(\gamma=1.4)$ was conducted on the same grid with $701 \times 201$ nodes. Parameters of the free stream flow
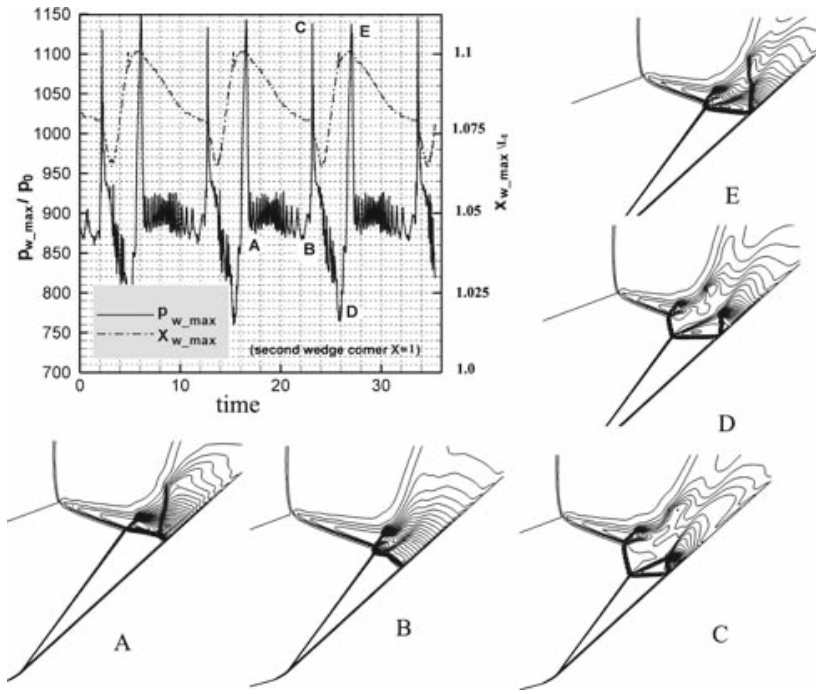

Fig. 11 Variation of the maximum pressure along the wedge surface (solid line), its location (dash line) and the shock flow patterns for an ideal gas flow $\left(\gamma=1.4, M_{\infty}=9, \theta_{1}=15^{\circ}, \theta_{2}=42.35^{\circ}\right)$

and the computational domain were set as follows, $M_{\infty}=9$, $\theta_{1}=15^{\circ}, \theta_{2}=42.35^{\circ}$ according to the computational setup in Ben-Dor's work [5] where oscillation was first observed. The variation of the maximum pressure along the wedge surface and its location were plotted in Fig. 11. The shock flow patterns at several significant instants were also given in the same figure. Qualitatively similar with phenomenon described in Sect. 3.3, the pressure pulse, which is around $1,140 p_{\infty}$, occurs shortly before the seven-shock to six-shock transition or shortly after the six-shock to seven-shock transition. Further computations indicate that the self oscillations undergo inside a wedge angle range, $42^{\circ} \leq \theta_{2} \leq 43^{\circ}$. Therefore it cab be concluded that the different findings in Sect. 3.3 aren't due to the difference of computational resolution or scheme dependency, but due to the difference of gas model.

\subsection{Viscous effects}

Viscous dissipation can result in different flow topologies, and can possibly damp down the oscillation in the numerical simulations. Shock wave/boundary layer interactions can also alter the shock interaction patterns. It is a critical challenging problem to consider the viscous effects on the complete double wedge geometry. Numerical difficulties may arise due to the thin boundary layer and shock wave interactions. Even worse, a separation bubble in the vicinity of the wedge corner and separation shock-lets cause a series of complicated and unsteady flow phenomena $[6,7]$ that couple and intermix viscous and inviscid effects. To eliminate such difficulties and avoid associated complexity, the computational geometry was simplified, as shown in Fig. 12. The first wedge is treated as an inviscid wall connecting the second wedge with 


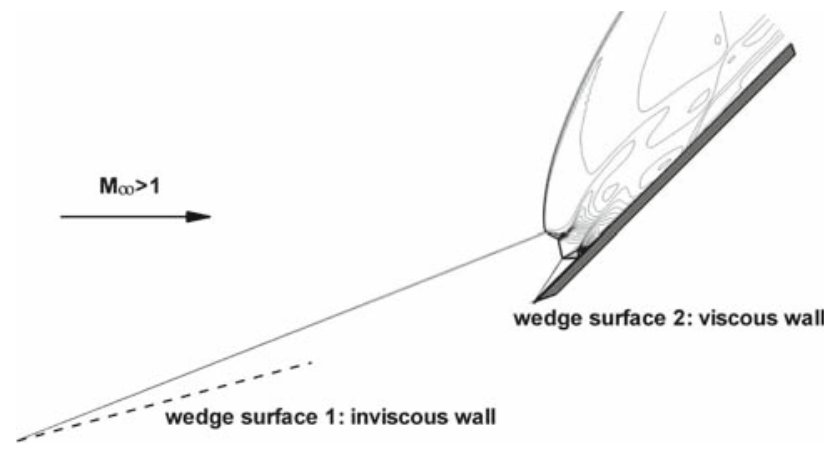

Fig. 12 Sketch of the computational geometry for consideration of the viscous effect on the self-oscillation

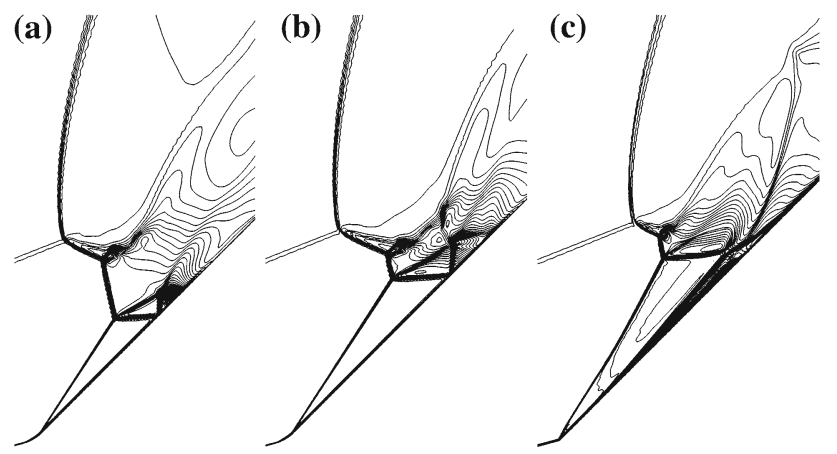

Fig. 13 Comparison between inviscid $\mathbf{a}, \mathbf{b}$ and viscous $\mathbf{c}$ solutions $\left(M_{\infty}=7, \theta_{1}=15^{\circ}, \theta_{2}=45^{\circ}\right)$

a viscous surface. In the following simulations, mesh of the same total nodes, i.e. $701 \times 201$, was used while being clustered more near the surface to ensure $y^{+}$for the first grid point off the second wedge less than 1.36.

A comparison between the inviscid and laminar viscous solutions is shown in Fig. 13. The unsteady movements of the bow shock wave were not found in the present viscous simulation, and instead, a steady shock flow pattern was achieved as shown in Fig. 13c. The location of the triple shock structure is close to the right extreme location of the Type $\mathrm{V}$ shock/shock interaction structure from the Euler solution, as shown in Fig. 13b. However, the leading shock starting from the second wedge is no longer straight due to the effect of the boundary layer. The reflected shock wave of the lower triple shock structure re-reflects over the second wedge, forming a separation bubble due to the SBLI. After the bubble apex, an expansion fan and a reattachment shock are then generated. It is also obvious that all of the contact discontinuities of the three triple points are smeared by viscous dissipation. This is likely part of the mechanism that damps down the oscillation induced by shock/shock interactions. As demonstrated in the experiments, the unsteady movement of the bow shock is quite small; it has been suggested that it is associated with unsteadiness in the reattachment region [7]. Therefore, the viscous effect on the unsteady oscillations remains unclear thus far. Further numerical or experimental studies will be necessary to solve this issue.

\section{Conclusions}

In this paper, the shock pattern oscillations induced by shock/shock interactions over double-wedge geometries in hypersonic flows were studied numerically by solving $2 \mathrm{D}$ inviscid Euler equations. A multi-species system with temperature-dependent thermodynamic properties was utilized. It was shown that the oscillation results in high-frequency fluctuations of heating and pressure loads over the second wedge surface. Different oscillations of shock flow patterns, such as seven-shock to seven-shock, seven-shock to sixshock, and seven-shock to seven-shock to six-shock transitions, were obtained according to different free stream flow Mach numbers and wedge angles. The numerical results also indicated that the critical wedge angle for transition from the steady to the oscillation solution is higher compared to the corresponding value in earlier numerical study in which perfect diatomic gas was used as the fluid medium. Viscous simulations indicated that the oscillation can be likely damped down due to viscous dissipation.

Acknowledgments This work was supported by Korea Research Foundation Grant No. KRF-2005-005-J09901.

\section{References}

1. Bertin, J.J., Cummings, R.M.: Fifty years of hypersonics: where we've been, where we're going. Prog. Aerosp. Sci. 39, 511-536 (2003)

2. Bertin, J.J., Cummings, R.M.: Critical hypersonic aerothermodynamic phenomena. Ann. Rev. Fluid Mech. 38, 129-157 (2006)

3. Edney, B.: Anomalous heat transfer and pressure distributions on blunt bodies at hypersonic speeds in the presence of an impinging shock. Rep. 115, The Aerospace Research Institute of Sweden, Stockholm (1968)

4. Olejniczak, J., Wright, W.J., Candler, G.V.: Numerical study of inviscid shock interactions on double-wedge geometries. J. Fluid Mech. 352, 1-25 (1997)

5. Ben-Dor, G., Vasilev, E.I., Elperin, T., Zenovich, A.V.: Self-induced oscillations in the shock wave flow pattern formed in a stationary supersonic flow over a double wedge. Phys. Fluids 15(12), L85L88 (2003)

6. Schrijer, F.F.J., Scarano, F., van Oudheusden, B.W.: Application of PIV in a Mach 7 double-ramp flow. Exp. Fluids 41, 353-363 (2006)

7. Schrijer, F.F.J., van Oudheusden, B.W., Dierksheide, U., Scarano, F.: Quantitative visualization of a hypersonic double-ramp flow using PIV and schlieren. In: 12th International Symposium on Flow Visualization, Göttingen (2006)

8. Burtschell, Y., Zeitoun, D.E.: Shock/shock and shock/boundary layer interactions in an axisymmetric steady laminar flow. Shock Waves 12, 487-495 (2003)

9. McBride, B.J., Zehe, M.J., Gordon, S.: NASA Glenn coefficients for calculating thermodynamic properties of individual species. NASA/TP 2002-211556, Glenn Research Center, Cleveland (2002) 
10. Svehla, R.A.: Transport coefficients for NASA Lewis chemical equilibrium program. NASA TM 4647, Lewis Research Center, Cleveland (1995)

11. Jiang, Z.L., Takayama, K., Chen, Y.S.: Dispersion conditions for non-oscillatory shock-capturing schemes and its applications. Comput. Fluid Dyn. J. 2, 137-150 (1995)

12. Jiang, Z.L.: On the dispersion-controlled principles for nonoscillatory shock-capturing schemes. Acta Mech. Sinica 20(1), $1-15$ (2004)

13. Jiang, Z., Takayama, K., Babinsky, H., Meguro, T.: Transient shock wave flows in tubes with a sudden change in cross section. Shock Waves 7(3), 151-162 (1997)

14. Jiang, Z.L., Takayama, K.: An investigation into the validation of numerical solutions of complex flowfields. J. Comput. Phys. 151(2), 479-497 (1999)
15. Jiang, Z.L.: Reliable validation based on optical flow visuallization for CFD simulations. Acta Mech. Sinica 19(3), 193-203 (2003)

16. Jiang, Z.L., Huang, Y.H., Takayama, K.: Shocked flows induced by supersonic projectiles moving in tubes. Comput. Fluids 33(7), 953966 (2004)

17. Hu, Z.M., Jiang, Z.L.: Wave Dynamic process in cellular detonation reflection from wedges. Acta Mech. Sinica 23(1), 33-41 (2007)

18. Hu, Z.M., Myong, R.S., Nguyen, A.T., Jiang, Z.L., Cho, T.H.: Numerical analysis of the flowfield in a supersonic COIL with an interleaved jet configuration and its effect on the gain distribution. Eng. Appl. Comput. Fluid Mech. 1(3), 207-215 (2007)

19. Hu, Z.M., Jiang, Z.L., Myong, R.S., Cho, T.H.: Numerical analysis of spatial evolution of the small signal gain in chemical oxygeniodine laser operating without primary buffer gas. Opt. Laser Technol. 40, 13-20 (2008) 